Z. klin. Chem. u. klin. Biochem.

9. Jg., S. 95-98, März 1971

\title{
Gaschromatographische Analyse des Kohlenhydratanteiles serologisch aktiver Glykoproteine
}

\author{
Von B. SAlfNer und G. UhLENBRUCK
}

Aus der Mediqinischen Universitätsklinik Köln-Lindenthal

(Direktor: Prof. Dr. R. Gross)

(Eingegangen am 23. November 1970)

Es wird die Analyse von Monosacchariden serologisch aktiver Glykoproteine mit Hilfe der Gaschromatographie beschrieben. Die A-Substanzen aus Pepton und Tubifex rivulorum sowie die Amnionmucoide von Rind und Schwein werden analysiert und miteinander verglichen.

Die Methode umfaßt Hydrolyse mit wasserfreier, methanolischer $\mathrm{HCl}, \mathrm{N}-$ Reacetylierung der Aminozucker, Uberführung der MethylGlykoside in Trimethylsilyl-Derivate und gaschromatographische Bestimmung.

\section{Analysis of the carbobydrate component of serologically active glycoproteins by gas-liquid-cbromatograpby}

The analysis of the monosaccharides of serological active glycoproteins using gas-liquid-chromatography is described. The A-substances from Peptone and from Tubifex rivulorum and the bovine and pig amnionmucoids were analysed and compared.

The method consists of hydrolysis with anhydrous methanolic $\mathrm{HCl}, \mathrm{N}$-reacetylation of the hexosamines, conversion of the methylglycosides to trimethylsilyl-derivatives and their determination by gas-liquid-chromatography.

Colorimetrische Bestimmungsmethoden von Monosacchariden aus Glykoproteinen sind zeitaufwendig und bergen voneinander unabhängig die Gefahr experimenteller Fehlerquellen. Es ist daher nicht verwunderlich, $\mathrm{da} B$ man auf der Suche nach weniger aufwendigen Methoden auf die Gaschromatographie (VerteilungsGaschromatographie) stieß, ein Verfahren, das sich bei der Analyse von Fetten und Aminosäuren ausgezeichnet bewährt hatte. Die Hauptschwierigkeiten bei der gaschromatographischen Analyse von Zuckern lagen in der Notwendigkeit, die an sich wenig flüchtigen Zucker in leichter flüchtige Derivate umzuwandeln und zweitens in der Vielzahl von Anomeren, deren Trennung eine große Trennleistung der Säule erfordert. Während das erste Problem inzwischen als gelöst angesehen werden kann $(13,14,16)$, bietet die Trennung der Zuckeranomeren mit gepackten Säulen noch Schwierigkeiten, die sich auf die absolute quantitative Analyse auswirken können.

Die vorliegende Arbeit befaßt sich mit der Anwendung der Gaschromatographie auf die quantitative Analyse des Zuckeranteiles von vier serologisch aktiven Mucoiden und dem Vergleich dieser Substanzen.

\section{Methodik}

Geräte

Gaschromatograph der Firma Packard, Frankfurt, mit GlasSpiralsäulen (185 cm I.änge, $5 \mathrm{~mm}$ Durchmesser), Temperaturprogrammierung und Flammenionisations-Detektor.

Rotationsverdampfer Rotavapor-R der Firma Büchi.

\section{Chemikalien}

Essigsäureanhydrid p. a., Merck, Art.-Nr. 42

Hexamethyldisilazan purum, Fluka AG
n-Hexan rein, Merck, Art.-Nr. 4368

Kaliumhydroxid Plätzchen rein, Merck, Art.-Nr. 5033

Methanol p. a., Merck, Art.-Nr. 6009

Pyridin rein, Merck, Art.-Nr. 7461

Schwefelsäure 95-97\% p. a., Merck, Art.-Nr. 731

Silbercarbonat, Riedel-de Haën AG, Seelze-Hannover

Trimethylchlorsilan reinst, Serva, Heidelberg

\section{Arbeitsvorschrift}

\section{Gevinnung der Mucoide}

Die Mucoidherstellung wurde in früheren Arbeiten beschrieben und soll hier nur kurz zusammengefaßt werden.

A-Substanz aus Pepton S der Firma Brunnengräber, Lübeck (Methode nach Weșrphar): Phenol-NaCl-Extraktion, Dialyse der wäßr. Phase gegen dest. Wasser, Lyophilisierung (1).

A-Substanz aus Tubifex rivulorum (Schlammröhrenwurm): Wässerung der Tiere in Leitungswasser, zentrifugieren, homogenisieren mit etwas $\mathrm{NaCl}$, Phenol-Extraktion in der Kälte, Dialyse der wäßr. Phase gegen dest. Wasser, Lyophilisierung (2).

Amnionmucoide: gleiche Teile Flüssigkeit und 90 proz. Phenol in der Kälte extrahieren, Dialyse der wäßr. Phase gegen dest. Wasser, Lyophilisierung (3).

\section{Hydrolyse}

Die Abspaltung der Monosaccharide von den Glykoproteinen erfolgte durch saure Hydrolyse mit wasserfreier methanolischer $\mathrm{HCl}$. Methanol wurde redestilliert über Magnesiumspänen (Kochen im Rückfluß für $2 \mathrm{Stdn}$ ). Zur Herstellung der methanolischen $\mathrm{HCl}$ wurde 97 proz. $\mathrm{H}_{2} \mathrm{SO}_{4}$ tropfenweise auf Natriumchlorid gegeben und die entstehende $\mathrm{HCl}$ nach Reinigung über Waschflaschen in Methanol aufgefangen. Dic Hydrolyse wurde mit $1 \mathrm{~N}$ methanolischer $\mathrm{HCl}$ bei $75^{\circ}$ unter Sauerstoffausschluß in dickwandigen, versiegelten Glasampullen durchgeführt. Die Hydrolysedauer betrug 6,12 und $24 \mathrm{Stdn}$. Die eingewogenen Substanzmengen enthielten 10 bis $20 \mathrm{mg}(5-10 \mathrm{mg} / \mathrm{m} /$ methanolischer $\mathrm{HCl})$.

\section{Reacetylierung der Aminozucker}

Die $\mathrm{HCl}$ wurde mit $\mathrm{Ag}_{2} \mathrm{CO}_{3}$ neutralisiert, zentrifugiert, det Überstand in cin Schliffgläschen überführt und rasch am Rotationsverdampfer im Vakuum bis zur Trockene cingedampft. Das Sediment 
Tab. 1

Relative Retentionszeiten von persilylierten Methyl-Glykosiden, bezogen auf Mannose. (Säule: OV $173 \%$ auf Gas-Chrom Q)

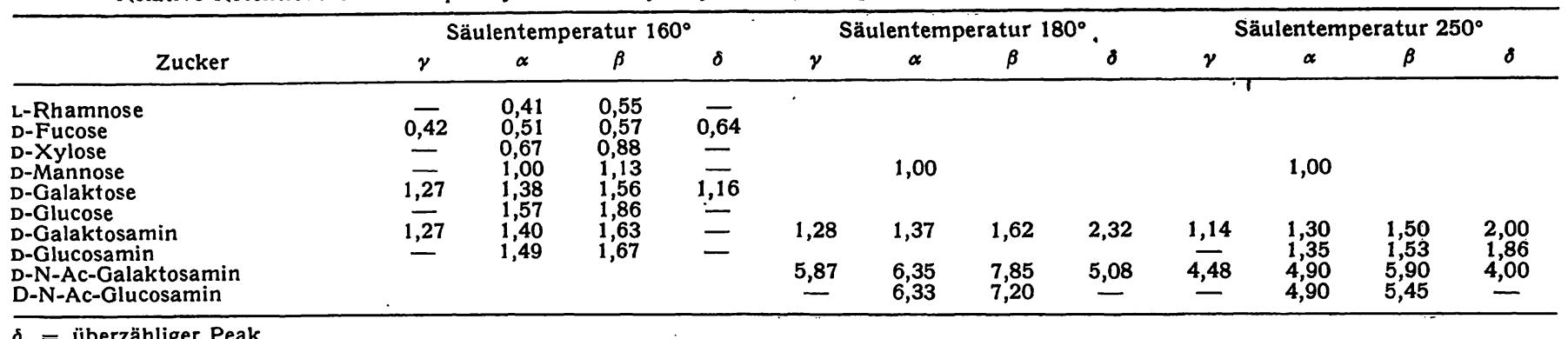

$\delta=$ überzähliger Peak

wurde mit wasserfreiem Methanol ausgewaschen und der Uberstand ebenfalls eingedampft. Nach Lagerung im Exsikkator über $\mathrm{P}_{2} \mathrm{O}_{5}$ für etwa $12 \mathrm{Stdn}$. wurden die Proben mit einem Gemisch von redestilliertem Essigsäureanhydrid/Methanol $(1: 3 \mathrm{v} / \mathrm{v})$ versetzt und bei Raumtemperatur 12 Stdn. stehengelassen.

\section{Silylierung}

Nach erneutem Eindampfen bis zur Trockene wurden die Monosaccharide in Trimethylsilylderivate überführt. Wir folgten dabei der Vorschrift von Gheorghiu und Oette $(4,11,17)$. Die Proben wurden unter $\mathrm{N}_{2}$ mit etwa $0,5 \mathrm{~m} /$ Silylierreagenz $/ 10 \mathrm{mg}$ Substanz versetzt und $30 \mathrm{Min}$. unter wiederholtem Schütteln bei Raumtemperatur stehengelassen. Nach sorgfältigem Abblasen der flüchtigen Anteile mit $\mathrm{N}_{2}$ vurde die Substanz in einer definierten Menge Hexan gelöst.

\section{Gaschromatographische Auftrennung}

Säulenfüllungen: Äthylenglycolsuccinat (EGS) 2,5\% auf Chromosorb G (80-100 mesh) (Applied Science Laboratories, Pa. USA über Serva Heidelberg).

SE 30 1\% auf Chromosorb G (80-100 mesh) (Serva Heidelberg). OV 17 3\% (Methylsilicone mit 50\% Phenyl-Substitution) auf GasChrom Q (100-120 mesh) (Serva Heidelberg).

Säulentemperatur: Tc $100^{\circ}$, Ti $160^{\circ}$ für EGS-Säulen

Tc $160^{\circ}$, Ti $220^{\circ}$ für OV 17 und SE 30-Säulen.

Es wurde je nach Fragestellung mit oder ohne Temperaturprogrammierung gearbeitet. Die Temperatur des Inlets betrug $240^{\circ}$, die des Outlets $230^{\circ}$, des Detektors $230^{\circ}$.

Als Trägergas wurde Argon $99,99 \%$ (Fa. Messer-Griesheim/Köln) verwendet. Bei einem Gasdurchfluß von $40-80 \mathrm{ml} / \mathrm{Min}$. betrug der Gasdruck 15-25 pound's/square inch.

Spannung: $150 \mathrm{~V}$, Empfindlichkeit $10^{-8} \times 3 / 10^{-8} \times 1$.

$1-8 \mu l$ in Hexan gelöste Substanz wurden mit Hilfe einer Hamiltonspritze auf die Säule gespritzt.

Auswertung: Die qualitative Analyse wurde an Hand einer Standardmischung und mit Hilfe der relativen Retentionszeiten durchgeführt.

Zur quantitativen Analyse wurde der Substanz ein innerer Standard (L-Rhamnose 5-10\% der Substanzmenge) vor bzw. unmittelbar nach der Hydrolyse zugegeben. Berechnet wurden die zeichnerisch in Dreiecke umgewandelten Peakflächen nach Höhe $_{\mathbf{D}} \cdot 0,5$ Basis (5).

\section{Neuraminsäurebestimmung}

N-Acetyl-Neuraminsäure wurde getrennt von der Gaschromatographie nach der Methode von BraL, modifiziert nach BöHM und Mitarbeiter (6), photometrisch bestimmt (ohne die Verwendung von Trichloressigsäure).

\section{Ergebnisse}

Tabelle 1 zeigt eine Zusammenstellung der relativen Retentionszeiten der persilylierten Methylglycoside, bezogen auf die unpolare Säule OV 17. Der Vorteil dieser Säule liegt in der vollkommenen Symmetrie der Peakform, es tritt keinerlei Tailing auf.

Eine vollständige Trennung der Zuckeranomeren konnten wir nicht erreichen. Für die Peaks von $\alpha$-Galaktose/ $\alpha$-Galaktosamin, $\beta$-Galaktose / $\alpha$-Glucosamin sowie $\beta$-Galaktoasmin / $\beta$-Glucosamin traten Überlappungen auf. Die quantitative Analyse war mit Hilfe des günstigen Anomerenverhältnisses (Tab. 2) bei der wasserfreien Methanolyse möglich.

Tab. 2

Anomerenverhältnis persilylierter Methyl-Glykoside (Säule: OV $173 \%$, Temperatur $160^{\circ}$ )

\begin{tabular}{|c|c|c|c|c|}
\hline Zucker & $\gamma$ & $\alpha$ & $\boldsymbol{\beta}$ & $\delta$ \\
\hline $\begin{array}{l}\text { Rhamnose } \\
\text { Fucose } \\
\text { Xylose } \\
\text { Mannose } \\
\text { Galaktose } \\
\text { Glucose } \\
\text { Galaktosamin } \\
\text { Glucosamin } \\
\text { N-Acetyl-Galaktosamin } \\
\text { N-Acetyl-Glucosamin }\end{array}$ & $\begin{array}{l}\frac{}{2,50} \\
\frac{\overline{3}}{3,55} \\
\frac{8,10}{4,20}\end{array}$ & $\begin{array}{l}98,50 \\
71,50 \\
97,00 \\
92,50 \\
64,75 \\
1,60 \\
77,95 \\
3,70 \\
78,80 \\
11,20\end{array}$ & $\begin{array}{r}1,50 \\
21,20 \\
3,00 \\
7,50 \\
25,85 \\
98,40 \\
9,54 \\
96,30 \\
11,90 \\
88,80\end{array}$ & $\begin{array}{l}\overline{4,90} \\
\overline{5,78} \\
\overline{4,41} \\
\overline{5,10}\end{array}$ \\
\hline
\end{tabular}

$\delta=$ überzähliger Peak

Mit der in der Literatur $(7,8,15)$ angegebenen Methode konnten wir keine vollständige N-Reacetylierung der Hexosamine erreichen. Aụch bei Temperaturerhöhung auf $40^{\circ}$, Erhöhung der Konzentration des Essigsäureanhydrids sowie Verlängerung der Acetylierungsdauer auf 24 Stdn., waren trotz streng wasserfreien Arbeitens noch freie Aminozucker vorhanden.

Der Einfluß der HCl-Konzentration und der Hydrolysedauer auf die Monosaccharidbestimmung ist am Beispiel der A-Substanz aus Pepton (Tab. 3) aufgezeigt. Die Maximalwerte liegen bei einer Hydrolysedauer von 12 Stdn. Die größte Ausbeute an Hexosaminen erhiel-

Tab. 3

Einfluß der $\mathrm{HCl}-$ Konzentration und der Methanolysedauer auf die Monosaccharidbestimmung von A-Substanz aus Pepton. (Angegebene Werte in \% Peakfläche gegenüber Peakfläche des internen Standards $=100 \%$ )

\begin{tabular}{|c|c|c|c|c|c|c|c|c|c|}
\hline Zucker & 6 Stdn. & $\begin{array}{l}0,5 \mathrm{~N} \mathrm{HCl} \\
12 \mathrm{Stdn} .\end{array}$ & 24 Stdn. & 6 Stdn. & $\begin{array}{c}1 \mathrm{~N} \mathrm{HCl} \\
12 \mathrm{Stdn} .\end{array}$ & 24 Stdn. & 6 Stdn. & $\begin{array}{l}2 \mathrm{~N} \mathrm{HCl} \\
12 \mathrm{Stdn} .\end{array}$ & 24 Stdn. \\
\hline $\begin{array}{l}\text { Fucose } \\
\text { Mannose } \\
\text { Glucose } \\
\text { Galaktose } \\
\text { Galaktosamin }\end{array}$ & $\begin{array}{r}28,4 \\
17,2 \\
8,5 \\
31,1 \\
51,0\end{array}$ & $\begin{array}{l}36,8 \\
18,3 \\
10,7 \\
33,9 \\
55,7\end{array}$ & $\begin{array}{r}29,7 \\
19,0 \\
9,6 \\
33,0 \\
54,4\end{array}$ & $\begin{array}{r}27,5 \\
17,0 \\
8,4 \\
32,4 \\
56,0\end{array}$ & $\begin{array}{r}35,3 \\
18,3 \\
9,8 \\
32,8 \\
58,8\end{array}$ & $\begin{array}{r}29,1 \\
17,6 \\
9,2 \\
33,9 \\
59,2\end{array}$ & $\begin{array}{l}36,3 \\
16,8 \\
10,7 \\
36,2 \\
61,5\end{array}$ & $\begin{array}{r}28,4 \\
15,4 \\
9,7 \\
33,3 \\
64,4\end{array}$ & $\begin{array}{r}27,1 \\
15,0 \\
9,7 \\
30,7 \\
62,1\end{array}$ \\
\hline
\end{tabular}


ten wir mit $2 \mathrm{~N}$ methanolischer $\mathrm{HCl}$, die hierbei auftretenden zusätzlichen Peaks störten jedoch die quantitative Analyse.

Bei Hydrolyse mit $1 \mathrm{~N}$ methanolischer $\mathrm{HCl}$ traten bei den Zuckern Fucose, Galaktose und den Hexosaminen je ein zusätzlicher Peak auf. Die Fläche machte jedoch weniger als $5 \%$ der Gesamtpeakfläche aus, eine Beeinträchtigung der quantitativen Auswertung war daher kaum vorhanden.

Tab. 4

Ansprechbarkeit des Flammenionisationsdetektors (Binäre Mischungen von Testzuckern, Säule: OV $173 \%$ )

\begin{tabular}{lccc}
\hline Zucker & $\begin{array}{c}\text { eingewogene } \\
\text { Menge (mg) }\end{array}$ & $\begin{array}{c}\text { wiedergefundene } \\
\text { Menge (mg) }\end{array}$ & $\begin{array}{c}\text { Differenz } \\
\%\end{array}$ \\
\hline Rhamnose & 2,1 & 2,138 & $+1,78$ \\
Mannose & 2,15 & 2,117 & $\pm 1,56$ \\
Glucose & 3,0 & 2,813 & $=6,66$ \\
Glucose & 6,1 & 5,74 & $=6,27$ \\
Galaktose & 3,1 & 2,79 & $=11,1$ \\
N-Acetyl-Galaktosamin & 2,2 & 2,098 & $-4,86$ \\
\hline
\end{tabular}

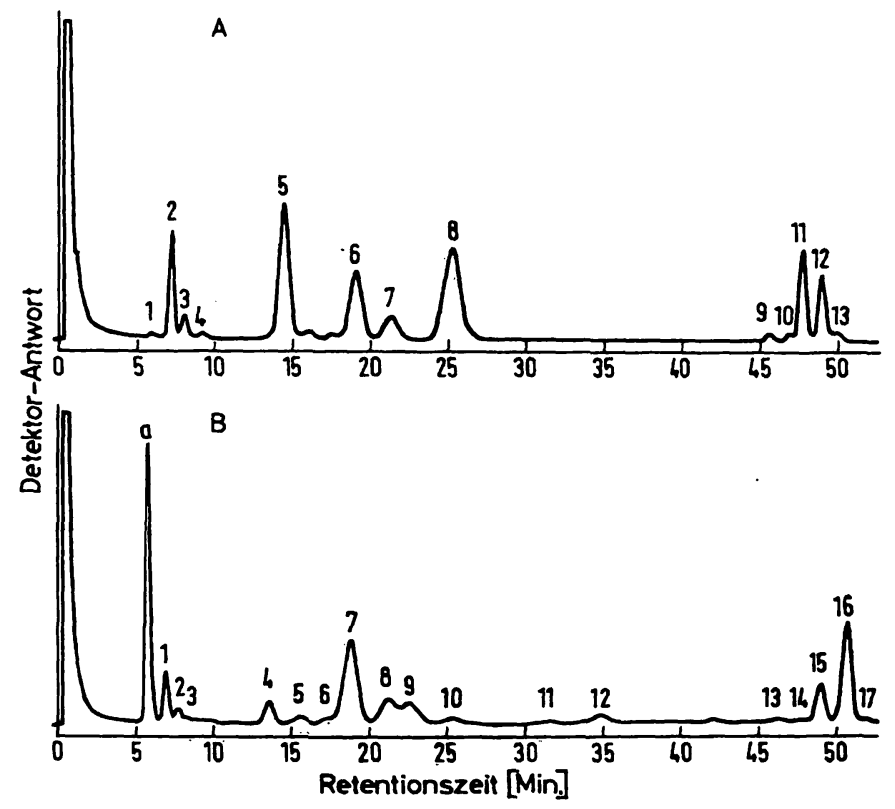

Abb. 1 a

Gaschromatogramm eines Testgemisches. Bedingungen: ov $173 \%$, $160-210^{\circ}, 15^{\circ} / \mathrm{Min}$. ab Eluierung der Glucose. $E=10^{-8} \times 1,1,25 \mathrm{~cm} /$ $M i n$
$\beta$-Glucose

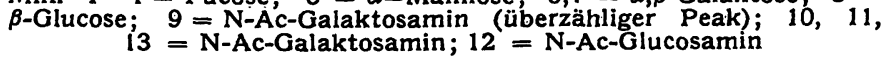

Abb. 1 b

Gaschromatogramm der A-Substanz aus Pepton. Bedingungen: wie bei 1 a. $a=$ Rhamnose (interner Standard); $1-3=$ Fucose; $4=\alpha-$ Mannose; $5=\beta$-Mannose + Galaktose (überz. Peak); $6=\gamma$-Galaktose; $7=\alpha-$ Galaktose $+\alpha$-Galaktosamin; $8=\beta$-Galaktose $+\alpha-$ Glucosamin; $9=\beta$-Galaktosamin $+\beta$-Glucosamin; $10=$ Glucose; 11 = Galaktosamin (überz. Peak); $12=$ Glucosamin (überz. Peak); $13=\mathrm{N}-\mathrm{Ac}-$ Galaktosamin (überz. Peak); 14,15,
laktosamin; $16=\mathrm{N}$-Ac-Glucosamin
Die Linearität der Detektoransprechbarkeit geht aus Tabelle 4 hervor. Untersucht wurden binäre Mischungen von Testzuckern.

Abbildung 1 a zeigt das Gaschromatogramm eines Testgemisches und $1 \mathrm{~b}$ das Gaschromatogramm der A-Substanz aus Pepton.

In Tabelle 5 sind die Resultate der quantitativen Analyse der untersuchten Substanzen zusammengefaßt. Die Analyse der A-Substanz aus Tubifex rivulorum ergab große Mengen von Glucose. Es handelt sich dabei um eine Verunreinigung von glucosehaltigen Polysacchariden, die während der Aufarbeitung dieser Würmer mit in die Wasserphase gelangen (2). Die unverhältnismäßig große Glucosemenge ließ durch Überlagerung eine exakte Bestimmung der Monosaccharide, insbesondere eventuell vorhandener Galaktose, nicht zu.

Das molare Verhältnis der Monosaccharide ist in Tabelle 6 dargestellt.

Tab. 6

Molares Verhältnis der Monosaccharide einiger serologisch aktiver Glykoproteine (bezogen auf Fucose)

\begin{tabular}{|c|c|c|c|c|}
\hline Zucker & $\begin{array}{l}\text { Rinder- } \\
\text { amnion- } \\
\text { mucoid }\end{array}$ & $\begin{array}{l}\text { Schweine- } \\
\text { amnion- } \\
\text { mucoid }\end{array}$ & $\begin{array}{c}\text { A-Substanz } \\
\text { aus } \\
\text { Pepton }\end{array}$ & $\begin{array}{l}\text { A-Substanz } \\
\text { aus Tubi- } \\
\text { fex riv. }\end{array}$ \\
\hline $\begin{array}{l}\text { Fucose } \\
\text { Xylose } \\
\text { Mannose } \\
\text { Galaktose } \\
\text { Glucose } \\
\text { N-Ac-Galaktosamin } \\
\text { N-AC-Glucosamin }\end{array}$ & $\begin{array}{l}2 \\
1,5 \\
4 \\
8 \\
3 \\
3 \\
7\end{array}$ & $\begin{array}{r}2 \\
1 \\
2 \\
8 \\
1 \\
6 \\
14\end{array}$ & $\begin{array}{l}\frac{2}{1} \\
4 \\
0,5 \\
3 \\
6,5\end{array}$ & $\begin{array}{l}2 \\
1,5 \\
2 \\
? \\
? \\
3 \\
1,5\end{array}$ \\
\hline
\end{tabular}

Der Neuraminsäuregehalt des Rinderamnionmucoids betrug $10,1 \%$, der des Schweineamnionmucoids $7,0 \%$. Beide A-Substanzen enthielten keine Neuraminsäure.

\section{Diskussion}

Serologisch aktive Glykoproteine spielen eine große Rolle als Träger von Blutgruppeneigenschaften und anderen heterophilen Rezeptoren von Kohlenhydratnatur (Phytagglutininrezeptoren, Virusrezeptoren) (9). Die vorliegende Arbeit zeigt am Beispiel von vier serologisch wirksamen Mucoiden die gaschromatographische Analyse und den Vergleich der Heterosaccharidketten dieser Glykoproteine.

Bei der quantitativen Analyse von KohlenhydratEiweißverbindungen treten mehrere methodische Probleme auf:

Tab. 5

Analyse des Monosaccharidgehaltes einiger serologisch aktiver Glykoproteine (Angabe in \% der gesamten Substanzmenge unter Einbeziehung des Korrekturfaktors für den FID)

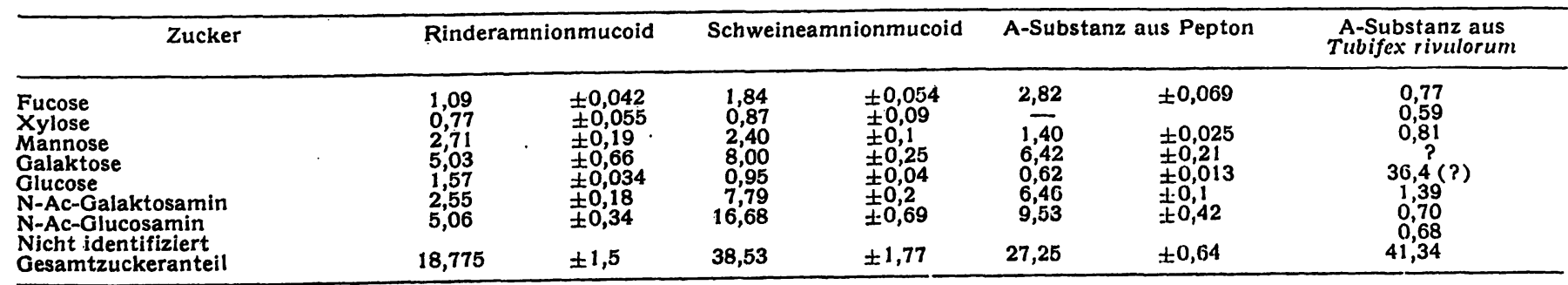


Eine vollständige Trennung der Zuckeranomeren kann mit gepackten Säulen nicht erreicht werden, da die NReacetylierung der Hexosamine und damit die Verlängerung der Retentionszeit dieser Zucker nur ungenügend gelingt. Dadurch wird eine Einbeziehung des Anomerenverhältnisses in die quantitative Berechnung erforderlich. Die saure, wasserfreie Methanolyse unter relativ milden Bedingungen birgt die Gefahr der unvollständigen Zuckerfreisetzung, besonders der Hexosamine (10), und hat den Nachteil zusätzlich auftretender Peaks. Wir zogen jedoch die Methanolyse einer Hydrolyse mit wäßr. $\mathrm{HCl}$ vor, da Methylglykoside durch ein günstigeres Anomerenverhältnis eine bessere Berechnung der Zucker gewährleisten, die Dekomposition der schon freigesetzten Zucker bei der verwandten Säurestärke geringer ist (11) und eine Wechselwirkung der Zucker mit den Aminosäuren des Glykoproteins vermieden wird (7). Eine mathematische Berechnung von Hydrolyse-Korrekturfaktoren, wie sie von GHEORGhiv, Oetre und BaumanN (12) für die Hydrolyse mit wäßr. $\mathrm{HCl}$ angegeben wird, haben wir aus dem Grunde nicht durchgeführt, weil die Gefahr von Fehlbestimmungen bei der Methanolyse unter den verwendeten Bedingungen mehr in der unvollständigen Freisetzung der Hexosamine als in der Dekomposition der Zucker liegt. Wir haben deshalb in Parallelversuchen die Methanolysedauer variiert $(6,12,24 \mathrm{Stdn}$.). $\mathrm{Da}$ wir die Möglichkeit von geringen Verunreinigungen unserer Ausgangssubstanzen nicht mit Sicherheit ausschlieBen können, halten wir dieses Vorgehen in bezug auf unsere Fragestellung für ausreichend.
Ein Vergleich der A-Substanzen ist nur beschränkt möglich, da die quantitative Analyse des Glykoproteins aus Tubifex rivulorum unzureichend ist. A-Substanz aus Pepton enthält im Unterschied zu A-Substanz aus Tubifex keine Xylose, Tubifex enthält einen nicht identifizierten Zucker (wahrscheinlich Mannosamin). In beiden Substanzen findet sich als Ausdruck ihrer BlutgruppenA Spezifität N-Acetyl-Galaktosamin.

Die Unterschiede der beiden Amnionmucoide sind quantitativer Art. Über den hohen Gehalt an Hexosamin und N-Acetyl-Neuraminsäure wurde schon früher (3) berichtet. Überraschend ist der relativ hohe Prozentsatz des Rinderamnionmucoids an Mannose. Wir nahmen deshalb an, daß das Rinderamnionmucoid nach Behandlung mit Neuraminidase PHA ${ }^{1}$ )-Aktivität besitzen könnte. Diese Vermutung liẹ sich jedoch im Hemmversuch nicht bestätigen.

Die beschriebene gaschromatographische Analyse von serologisch aktiven Glykoproteinen hat sich als zeitsparende Methode zu quantitativen Bestimmung von Monosacchariden bewährt und läßt sich, bezogen auf unsere Fragestellung, mit genügender Sicherheit durchführen.

Die Untersuchungen wurden durch Mittel der Deutschen Forschungsgemeinschaft gefördert.

Wir möchten ganz besonders Herm Priv.-Doz. Dr. K. Oetre für die großzügige Unterstützung und seinen Rat bei der Durchführung der Arbeit unseren herzlichen Dank aussprechen.

1) PHA = Rezeptor für Phytagglutinin aus Phaseolus vulgaris.

\section{Literatur}

1. Uhlenbruck, G., I. Sprenger und G. I. Pardoe, Z. Immunitforsch., Jena 140, 496 (1970). - 2. UHLENBRUCK, G., U. REIFENBERg und O. Prokop, Experientia, Basel 25, 1180 (1969). 3. Lambotte, R. und G. UhIEnbruck, Nature (London) 212, 290 (1966). - 4. Gheorghiu, Th. und K. Oetre, J. Chromatog., 48, 430 (1970). - 5. KAISER, R., Chromatographie in der Gasphase; B. I. Hochschultaschenbücher Bd. 92/92a, Bibliographisches Institut, Mannheim (1969). - 6. BöHM, P., St. Dauber und L. Baumeister, Klin. Wschr. 32, 289 (1954). - 7. Clanip, J. R., G. Dawson und L. Hough, Biochim. biophysica Acta (Amsterdam) 148, 342 (1967). - 8. Sweeley, C. C. und B. Walker, Analytic. Chem., 36, 1461 (1964). - 9. Prokop, O. und G. UhlenbruCK, Lehrb. menschl. Blut- und Serumgruppen, Edition Leipzig (1966).
10. Neuberger, A. und R. D. Marshall, in A. Gottschalk Glycoproteins, Elsevier Publ. Comp. Amsterdam (1966). - 11. Sweeley, C. C., R. Bentley, M. Makita und W. W. Wells, J. Amer. chem. Soc. 85, 2497 (1963). - 12. Gheorghiv, Th., K. Oetre und V. BaumanN, Z. Naturforsch., 25b, 829 (1970). 13. Bentley, R., C. C. Sweelex, M. Makita und W. W. Wells, Biochem. Biophys. Res. Commun., 11, 14 (1963). - 14. Brsнop, C. T., Meth. Biochem. Analysis, 10, 1 (1962). - 15. Silkinson, G. und J. V. WheElock, J. Dairy Res., 37, 113 (1970). - 16. OAtes, M. D. G. und J. Schrager, Biochem. J., 97, 697 (1965). - 17. Hedglex, E. J. und W. G. Overend, Chem. Ind. (London) 378 (1960).
Prof. Dr. G. Uhlenibruck Abteilung Immunbiologie der Medizinischen Klinik 5 Köln 41

Kerpener Str. 15 